\title{
A Comparison of Methods for Estimating Reynolds Stress from ADCP Measurements in Wavy Environments
}

\author{
ANTHONY R. KIRINCICH \\ Woods Hole Oceanographic Institution, Woods Hole, Massachusetts \\ JOHANNA H. ROSMAN \\ Institute of Marine Sciences, University of North Carolina at Chapel Hill, Morehead City, North Carolina
}

(Manuscript received 3 January 2011, in final form 28 June 2011)

\begin{abstract}
Turbulent Reynolds stresses are now routinely estimated from acoustic Doppler current profiler (ADCP) measurements in estuaries and tidal channels using the variance method, yet biases due to surface gravity waves limit its use in the coastal ocean. Recent modifications to this method, including spatially filtering velocities to isolate the turbulence from wave velocities and fitting a cospectral model to the below-wave band cospectra, have been used to remove this bias. Individually, each modification performed well for the published test datasets, but a comparative analysis over the range of conditions in the coastal ocean has not yet been performed. This work uses ADCP velocity measurements from five previously published coastal ocean and estuarine datasets, which span a range of wave and current conditions as well as instrument configurations, to directly compare methods for estimating stresses in the presence of waves. The computed stresses from each were compared to bottom stress estimates from a quadratic drag law and, where available, estimates of wind stress. These comparisons, along with an analysis of the cospectra, indicated that spectral fitting performs well when the wave climate is wide-banded and/or multidirectional as well as when instrument noise is high. In contrast, spatial filtering performs better when waves are narrow-banded, low frequency, and when wave orbital velocities are strong relative to currents. However, as spatial filtering uses vertically separated velocity bins to remove the wave bias, spectral fitting is able to resolve stresses over a larger fraction of the water column.
\end{abstract}

\section{Introduction}

Acoustic Doppler current profiler (ADCP) velocity measurements have been used to estimate vertical profiles of turbulent momentum flux, described in a bulk sense as Reynolds stresses, via a technique known as the variance method (Lohrmann et al. 1990; Stacey et al. 1999a). These momentum fluxes transfer boundary forces through the water column and, in the coastal ocean, are critical to processes such as the across-shelf exchange of water masses. However, direct application of the variance method to estimate stress as well as shear production of turbulent kinetic energy is limited in this region because of a bias associated with surface gravity waves. While several modifications to the method have been developed to

Corresponding author address: Anthony R. Kirincich, 266 Woods Hole Road, Woods Hole, MA 02543.

E-mail: akirincich@whoi.edu eliminate wave biases, a detailed comparative analysis of these methods over the range of conditions present in the coastal ocean is required.

Because of their vertical profiling and long-term deployment capabilities, ADCPs have proven to be a useful tool for measuring turbulence, despite their increased noise levels relative to traditional turbulence sensors. Using the variance method, vertical profiles of Reynolds stresses have been successfully estimated from bursts of high-frequency (1-2-Hz sample rate) along-beam velocities (Stacey et al. 1999a,b; Rippeth et al. 2003; Williams and Simpson 2004; Nidzieko et al. 2006; Lu and Lueck 1999; Rippeth et al. 2002, 2003), allowing detailed work on momentum transfer and turbulence in tidal channels, rivers, and estuaries. However, in most coastal ocean environments surface gravity waves dominate the energy spectrum, as orbital velocities are orders of magnitude larger than those of turbulent eddies. With waves present, small but unavoidable errors in instrument tilt can result in stress 
biases much larger than the Reynolds stresses themselves (Trowbridge 1998).

Recently, modifications to the variance method have been proposed to improve stress estimates from ADCP measurements made in the presence of waves. The variance differencing technique, developed for acoustic Doppler velocimeters (ADVs) by Trowbridge (1998) and adapted for ADCPs by Whipple et al. (2005), differenced vertically separated along-beam velocities, using linear wave theory to account for vertical decay of wave velocities with depth, before applying the variance method. Rosman et al. (2008) introduced vertical (along beam) and horizontal (beam to beam) adaptive filtering (AF) to improve this method's performance for longer period waves, following a method developed for ADVs by Shaw and Trowbridge (2001). Finally, the cospectra fit (CF) method (Gerbi et al. 2008; Kirincich et al. 2010) estimates the stress by fitting an established semiempirical turbulence model to below-waveband cospectra. The CF method also provides an estimate of the horizontal length scale of the dominant stress-carrying eddies.

The datasets used to test each of these modifications differed in the ranges of wave period, wave height, and current speed observed as well as the configuration of the ADCP itself. Thus, there is considerable uncertainty about which of these methods would perform better under a given set of environmental conditions. More generally, it is still not known over what parameter range ADCP-based stress estimates can be accurately made using any of these methods. Enough datasets now exist to examine the performance of these methods across a range of flow conditions, enabling a detailed assessment of coastal ocean stress observations to be made.

The present work applies two methods-the vertical adaptive filtering method (Rosman et al. 2008) and the cospectra fit method (Kirincich et al. 2010) - to five datasets, having a range of wave and current characteristics, to determine the conditions under which successful Reynolds stress estimates can be made for each. These methods are applied to previously published ADCP velocity observations from Moorea, French Polynesia, and Santa Barbara, California (Rosman et al. 2008); a pair of wave-free estuarine deployments in Elkhorn Slough, California (Nidzieko et al. 2006); and the Martha's Vineyard Coastal Observatory (MVCO), Massachusetts (Kirincich et al. 2010). In the present study, the MVCO dataset is expanded to include the weakly stratified (wintertime) portion of a 1.5-yr record of velocity observations, a 30-day portion of which was used by Kirincich et al. (2010). Combined, these datasets span a range of possible wave and current conditions in the coastal ocean, and a range of instrument-based noise levels, allowing us to fully evaluate the performance of each method. For each dataset, independent estimates of near-bottom and, if available, near-surface stresses, as well as the vertical structure of stress are used to compare method performance. The benefits and limitations of the methods are assessed and the conditions under which each is most appropriate are discussed. It should be noted that methodological comparisons under varying levels of stratification, an equally important driver of stress biases, are beyond the scope of this paper as sufficient hydrographic observations were not available.

\section{Observations}

Observations from the five published datasets (Rosman et al. 2008; Kirincich et al. 2010; Nidzieko et al. 2006) are used to compare the results of stress-estimation methods under a variety of forcing and wave conditions as well as different instrument sampling schemes. An overview of the datasets is given in Table 1 and information about the current and wave conditions present are shown in Figs. 1 and 2. Complete details about each can be found in the original works.

Although all of the datasets used a Teledyne RD Instruments (T-RDI) 1200-kHz Workhorse ADCP, instrument configurations varied. The Moorea and Santa Barbara deployments conducted by Rosman et al. (2008) were made using T-RDI's fast-pinging sampling mode (mode 12), with ensemble sampling at $1 \mathrm{~Hz}$ using bin sizes of 0.25 and $0.5 \mathrm{~m}$, respectively. By averaging multiple subpings together, mode 12 sampling reduces uncertainties by a factor of $1 / \sqrt{N}$ (where $N$ is the number of subpings) relative to standard single-ping (mode 1) sampling. The January 2008 ADCP dataset from MVCO (http://mvcodata.whoi.edu) used in Kirincich et al. (2010) was expanded here to include all wintertime (i.e., weakly stratified) data from September 2007 through January 2009 , allowing a wider range of wind and wave conditions to be explored. The MVCO ADCP used T-RDI's standard single-ping (mode 1), sampled at $2 \mathrm{~Hz}$ using 1-m bins. Finally, the two instrument deployments made in the Elkhorn Slough, a wave-free estuary along the California coast, by Nidzieko et al. (2006) both sampled $0.25-\mathrm{m}$ bins at $1 \mathrm{~Hz}$, but with mode 12 and mode 1 sampling, respectively. The Elkhorn deployments were specifically made to compare the variance method stress results possible from the two sampling modes (Nidzieko et al. 2006) and are used here to compare the performance of the methods on datasets having different instrument noise levels without the complication of waves.

We include observations from both sampling types and variable bin thicknesses to evaluate performance differences between the methods in wavy environments that might be due to instrument sampling. Recent studies 
TABLE 1. Dataset sampling and uncertainties.

\begin{tabular}{|c|c|c|c|c|c|c|c|c|c|c|c|c|c|c|}
\hline \multirow[b]{3}{*}{ Deployment } & \multicolumn{8}{|c|}{ Data source and instrument setup } & \multicolumn{6}{|c|}{ Minimum uncertainties $\left(\times 10^{-5} \mathrm{~m}^{2} \mathrm{~s}^{-2}\right)$} \\
\hline & \multirow[b]{2}{*}{ Source } & \multirow{2}{*}{$\begin{array}{l}\text { T-RDI } \\
\text { Mode }\end{array}$} & \multirow[b]{2}{*}{ Length } & \multirow{2}{*}{$\begin{array}{c}\text { Sample } \\
\text { rate }\end{array}$} & \multirow{2}{*}{$\begin{array}{l}\text { Bin } \\
\text { size }\end{array}$} & \multirow{2}{*}{$\begin{array}{l}\text { Water } \\
\text { Depth }\end{array}$} & \multirow{2}{*}{$\begin{array}{l}\text { Bin } 1 \\
\text { height }\end{array}$} & \multirow[b]{2}{*}{ Orientation* } & \multicolumn{3}{|c|}{ Beams 1-2 } & \multicolumn{3}{|c|}{ Beams 3-4 } \\
\hline & & & & & & & & & VM & $\mathrm{AF}$ & $\mathrm{CF}$ & $\mathrm{VM}$ & $\mathrm{AF}$ & $\mathrm{CF}$ \\
\hline Santa Barbara & $\begin{array}{l}\text { Rosman } \\
\quad \text { et al. (2008) }\end{array}$ & 12 & 15 days & $1 \mathrm{~Hz}$ & $0.5 \mathrm{~m}$ & $10 \mathrm{~m}$ & $1.3 \mathrm{~m}$ & $28^{\circ}$ & & 1.6 & 7.8 & & 1.6 & 8.4 \\
\hline Moorea & $\begin{array}{l}\text { Rosman } \\
\quad \text { et al. (2008) }\end{array}$ & 12 & 13 days & $1 \mathrm{~Hz}$ & $0.25 \mathrm{~m}$ & $12 \mathrm{~m}$ & $1.0 \mathrm{~m}$ & $29^{\circ}$ & & 7.7 & - & & 0.6 & - \\
\hline MVCO & $\begin{array}{l}\text { Kirincich } \\
\quad \text { et al. (2010) }\end{array}$ & 1 & 1.5 years & $2 \mathrm{~Hz}$ & $1.0 \mathrm{~m}$ & $12 \mathrm{~m}$ & $3.3 \mathrm{~m}$ & $26^{\circ}$ & & 4.5 & 9.1 & & 4.4 & 8.9 \\
\hline Elkhorn M1 & $\begin{array}{l}\text { Nidzieko } \\
\text { et al. (2006) }\end{array}$ & 1 & 14 days & $1 \mathrm{~Hz}$ & $0.25 \mathrm{~m}$ & $7 \mathrm{~m}$ & $1.0 \mathrm{~m}$ & $1^{\circ}$ & 22.7 & 31.4 & 19.9 & 23.4 & 32.7 & 21.4 \\
\hline Elkhorn M12 & $\begin{array}{l}\text { Nidzieko } \\
\text { et al. (2006) }\end{array}$ & 12 & 14 days & $1 \mathrm{~Hz}$ & $0.25 \mathrm{~m}$ & $7 \mathrm{~m}$ & $1.0 \mathrm{~m}$ & $68^{\circ}$ & 2.1 & 2.6 & 7.7 & 2.1 & 3.0 & 8.3 \\
\hline
\end{tabular}

* Orientation of the 3-4 beam axis relative to the principal axis of the depth-averaged flow.

(Williams and Simpson 2004; Nidzieko et al. 2006) have shown that the lower noise floors possible using T-RDI's mode 12 sampling lead to improved stress estimates using the variance method, in comparison to mode 1 sampling. Additionally, ADCP velocities from smaller bin sizes will be more noisy compared to larger bins, although the effect is somewhat less than the noise differences between mode 1 and mode 12 sampling.

As stress calculations utilize the along-beam velocities, the orientation of the beam pairs relative to both the dominant flow and wave forcing directions are important to the quality of the stress results for each beam pair. For all three coastal ocean datasets (Table 1), the ADCP beam 3-4 axis was oriented close to the alongshelf direction (the direction of the dominant tidal forcing) while the 1-2 axis was oriented closer to the acrossshelf direction (the general direction of the incoming surface gravity wave field). In the Elkhorn Slough datasets, the mode-1 instrument (Elkhorn M1) was deployed with the 3-4 axis oriented along the axis of the channel while the mode 12 instrument (Elkhorn M12), about $30 \mathrm{~m}$ away, had the 1-2 axis oriented closer to the main channel axis.

The datasets span a range of flow strengths, velocity shears, wave heights, and wave periods. Tidal velocities dominated the along-shelf flow of the Santa Barbara and MVCO datasets, where standard deviations of the 20-min averaged velocities were $0.15-0.2 \mathrm{~m} \mathrm{~s}^{-1}$, while acrossshelf flows were much weaker. For the Santa Barbara and MVCO datasets, the velocity profiles had a strong bottom-boundary layer shape with maximum shear near the bed (Fig. 1). Near-bottom flows were much weaker at Moorea, as the instrument was located in a deep groove between reef spurs, thus the maximum shear occurred midwater column (Rosman et al. 2008). Calculated from the ADCP along-beam velocities using standard methods, estimated significant wave heights were generally less than
$1.5 \mathrm{~m}$ at Santa Barbara and Moorea but often greater than $1.5 \mathrm{~m}$ at MVCO (Fig. 1). Wave power spectra were centered at 10-12 s at Santa Barbara, 16-17 s at Moorea, and 6-9 s at MVCO. Waves at Santa Barbara and Moorea were more narrow-banded and dominated by swell while MVCO was dominated by shorter-period, broad-banded wind waves. In the Elkhorn Slough, tidally driven velocity standard deviations had moderate to small vertical shear that were maximum near the bottom, similar to Santa Barbara and MVCO, but twice the magnitude (Fig. 2).

For the near-surface stress comparisons shown for the MVCO dataset, wind velocities were measured at MVCO's nearby Air-Sea Interaction Tower (ASIT), located $1.2 \mathrm{~km}$ to the south-southwest in $17 \mathrm{~m}$ of water. Wind stresses were estimated following Large and Pond (1981) assuming neutral stability and rotated into the coordinate system defined by the ADCPs beam axes.

\section{Stress estimation from ADCP measurements}

\section{a. The variance method}

The basic methodology of the variance method (Lohrmann et al. 1990; Stacey et al. 1999a) uses the alongbeam velocities from a four-beam ADCP in the Janus configuration, typical of T-RDI ADCPs, to compute profiles of vertical Reynolds stress in the two horizontal directions. Theoretically, equations for the vertical stresses at a given depth can be derived from the along-beam velocity equations from the two opposing beam pairs. For example, the along-beam velocities for beams 1 and 2 of a bottommounted upward-looking ADCP can be written as

$$
\begin{aligned}
& u_{1}=-u \sin \theta-w \cos \theta \\
& u_{2}=u \sin \theta-w \cos \theta
\end{aligned}
$$



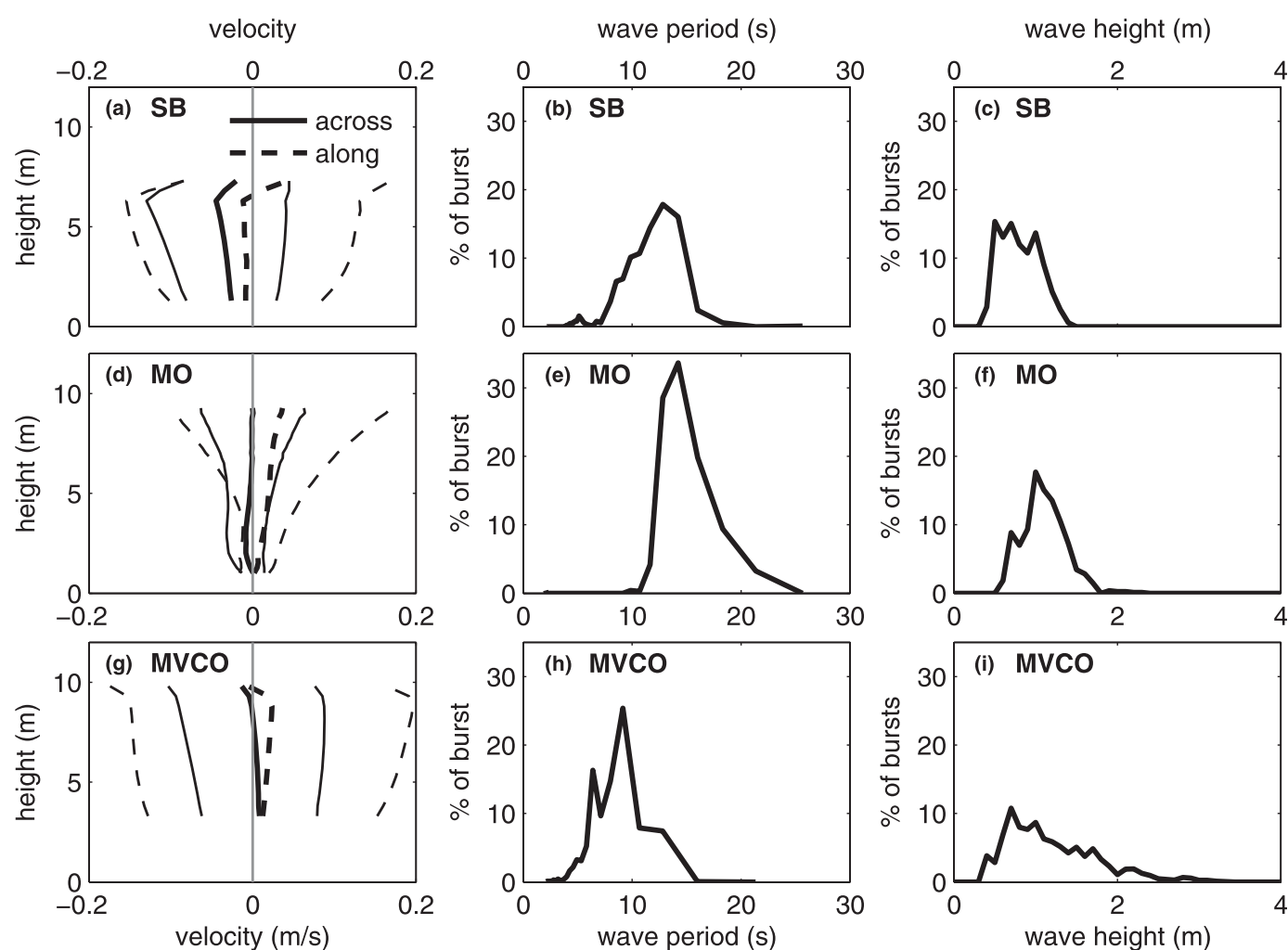

FIG. 1. Conditions during deployments at (a)-(c) Santa Barbara, (d)-(f) Moorea, and (g)-(h) the Martha's Vineyard Coastal Observatory. Shown are the burst-averaged velocity mean and mean \pm 1 std dev profiles in the along- (3-4 beam) and across- (1-2 beam) shelf directions [in (a),(d), and (g)], along with histograms of the dominant wave period [in (b),(e), and (h)] and significant wave height [in (c),(f), and (i)].

where $u_{1}$ and $u_{2}$ are the along-beam velocities in beams 1 and $2 ; u, v$, and $w$ are the $x, y$, and $z$ velocities in a righthanded coordinate system aligned with a plane defined by the beams (the beam $1-2$ axis); and $\theta=20^{\circ}$ is the angle of the beams away from vertical. If all fluctuations in a short (10-20 $\mathrm{min})$ burst around a mean velocity are due to turbulent motions only (the Reynolds decomposition), taking the variance of (1) and (2) and subtracting the two resulting equations gives an estimate of the 1-2 axis vertical Reynolds stress:

$$
\overline{u^{\prime} w^{\prime}}=\frac{\overline{u_{1}^{2}}-\overline{u_{2}^{2}}}{4 \cos \theta \sin \theta} .
$$

A similar equation exists for the 3-4 axis stress. In applying the variance method, it is assumed that turbulence is horizontally homogeneous; that is, turbulence statistics are the same at all four beam locations, and that turbulent statistics are stationary over the averaging interval.

\section{b. The adaptive fit method}

As described by Shaw and Trowbridge (2001) and modified for use with ADCPs by Rosman et al. (2008), the adaptive fit method uses least squares filtering to estimate the portion of along-beam velocity fluctuations in one depth bin that are coherent with the along-beam velocity in a second depth bin from the same ADCP beam. This coherent part, assumed to be due to waves, is subtracted from the velocity time series at the lower depth bin to isolate the remaining velocities, assumed to be turbulent fluctuations only. Correctly choosing the separation distance between the two along-beam locations is a critical part of performing the adaptive fit method properly. At smaller separations, the turbulent energy of the larger eddies will be subtracted along with the coherent wave energy. At larger values, the wave velocities become incoherent if waves are high frequency or if multiple wave frequencies are present, allowing wave energy to pass through the filter and bias stress estimates. Rosman et al. (2008) used the separation distance where the beam velocity variance versus bin separation curve reached a plateau (see their Fig. 11); finding vertical separations of 2 and $3 \mathrm{~m}$ for the Moorea and Santa Barbara datasets, respectively. A second technique, maximizing the correlation between the depth-averaged stress and a quadratic drag law in the "along-shelf" direction, used by Kirincich 

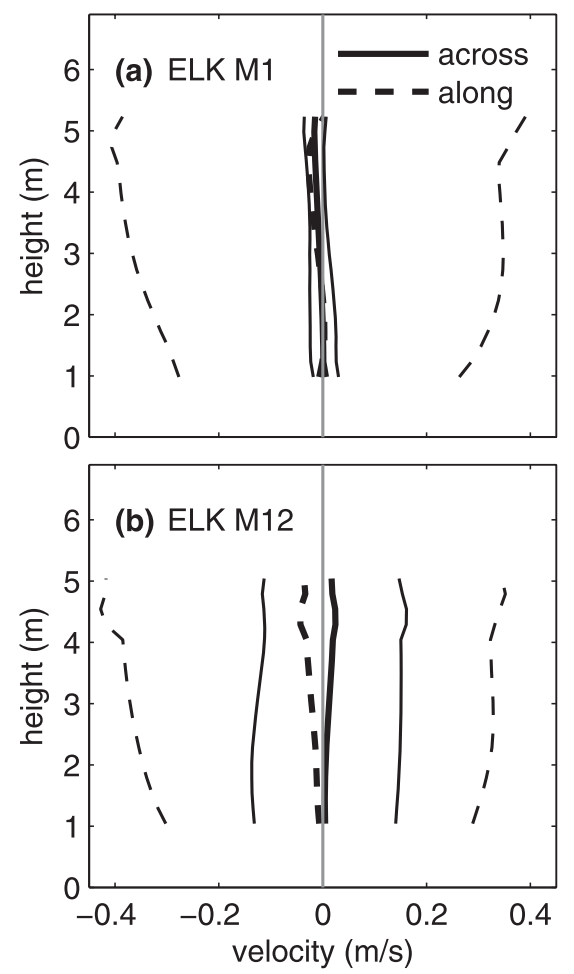

FIG. 2. Burst-averaged velocity mean and mean \pm 1 standard deviation profiles of the along- and across-channel directions for (a) Elkhorn M1 and (b) Elkhorn M12. Along-channel is defined as the 3-4 beam axis for Elkhorn M1, but the 1-2 beam axis for Elkhorn M12.

et al. (2010), gave similar results for Moorea and Santa Barbara as well as a separation distance of $3 \mathrm{~m}$ for the MVCO ADCP dataset. Following these techniques separations of $3 \mathrm{~m}$ were chosen for the wave-free datasets Elkhorn M1 and Elkhorn M12. However, there was some subjectiveness to determining the separation for the wave-free datasets based on these methods alone.

Uncertainty estimates for AF method stresses are based on the sum of the variance of squared along-beam velocities, reduced by the square of the denominator in Eq. (3) (Stacey et al. 1999a; Williams and Simpson 2004). This result is then adjusted by an additional parameter, on the order of $1-4$, to correct for the autocorrelation of the time series (Williams and Simpson 2004). However, following Rosman et al. (2008), the correlation correction was not implemented in the present study and thus the uncertainty estimates for the AF method shown represent a lower bound. In all AF method calculations, a lower limit to the matrix determinant, used to create the filter weights, was used to screen for ill-conditioned matrices.

\section{c. The cospectra fit method}

The CF method arrives at a stress estimate by considering the velocity cospectra of each burst time series at frequencies below those of surface gravity waves (Gerbi et al. 2008; Kirincich et al. 2010). The method fits a two-parameter, semiempirical model of the velocity cospectrum due to boundary layer turbulence (Kaimal et al. 1972) to the observed below-waveband cospectrum to estimate the total Reynolds stress and a "roll off" wavenumber $\left(k_{o}\right)$, a measure of the dominant length scale of turbulent fluctuations. To apply the CF method to ADCPbased along-beam velocities, a wave band cutoff frequency $\left(\omega_{\mathrm{wc}}\right)$ is defined as the lowest frequency at which a pseudospectrum of vertical velocity, derived from the spectrum of a collocated pressure sensor using linear wave theory, rises to $30 \%$ of the mean along-beam velocity spectrum. Using Taylor's frozen-flow hypothesis, the observed belowwaveband frequency cospectra from these point measurements of velocity are related to the wavenumber cospectra of the model via the mean velocity of the burst as $\omega=k|\mathbf{U}|$. Procedurally, the CF method uses a simple least squares fit between the cumulative integral-the ogive curve-of the observed and model cospectra at wavenumbers below the cutoff wavenumber $k_{\mathrm{wc}}$ to estimate the stress.

The drawback of the CF method is that it assumes the turbulent cospectrum has a defined structure centered about the roll-off wavenumber. However, the unsteady advection of turbulent eddies by wave velocities can alter the shape of the structure observed by aliasing lowerfrequency turbulent energy into the wave band (Lumley and Terray 1983; Trowbridge and Elgar 2001; Gerbi et al. 2008). This acts to decrease the total amount of turbulent energy seen below the wave band, and thus reduces the magnitude of a Reynolds stress estimated via the model fit of the below-waveband cospectrum, leading to a biased stress estimate. Using a threshold criterion developed by Gerbi et al. (2008), the ratio of the standard deviation of the wave orbital velocity ( $\left.\sigma_{\text {wave }}\right)$ to the burst-mean (or drift) velocity $\left(U_{d}=|\mathbf{U}|\right)$ must be less than $2\left(\sigma_{\text {wave }} / U_{d}<\right.$ 2) to limit bias to less than $15 \%$ of the estimated stress. This criterion serves to limit the conditions for which the CF method can be applied without significant error. Two additional criteria, applied after the stress is estimated, limit the results to times when the Kaimal et al. (1972) model is a good approximation to the observed cospectra, and exclude times where this is not the case. These criteria eliminate results where either the 1) estimated roll-off wavenumber was outside of the observed below-waveband cospectra or 2) less than $20 \%$ of the variance was explained by the model fit (Kirincich et al. 2010).

For the CF method, the uncertainty of the belowwaveband covariance, and by extension the total stress, are estimated using a nonparametric (Monte Carlotype) approach. Following Lu and Lueck (1999), (3) can be rewritten as a covariance of a sum and difference 
velocity time series. Lagging or shifting these time series relative to each other many (1000) times, by a random amount greater than the data-based decorrelation time scale (usually $\approx 30 \mathrm{~s}$ ), and computing the below-waveband covariance for each, builds a histogram of laggedcovariance estimates for each burst. The CF method stress uncertainty is defined as the standard deviation of the lagged-covariance histograms to match the uncertainty estimates of the variance method defined by Stacey et al. (1999a) and Williams and Simpson (2004).

\section{d. Calculation details}

Raw ADCP along-beam velocities from all datasets were carefully screened for data quality. For each 20-min burst of along-beam velocities, data at each depth level were screened and flagged for bad data characteristics, defined by the following: raw along-beam velocities greater than $2 \mathrm{~m} \mathrm{~s}^{-1}$, beam correlations (a measure of velocity precision) less than 90 counts, and beam intensities less than 60 counts. Velocities in all beams from a flagged bin were removed and linearly interpolated over in time. Interpolations of five or more consecutive points ( 2.5 or $5 \mathrm{~s}$ of data) were rejected and only continuous records longer than $10 \mathrm{~min}$ in which the fraction of interpolated pings was less than $10 \%$ were kept. These quality controlled data were used to estimate the burstmean horizontal velocities in the instrument coordinate system, the surface gravity wave statistics using standard ADCP-based techniques, and stresses via the methods described above. Prior to computing power spectra of the cleaned along-beam velocities, each 20 -min burst time series was detrended and tapered using a single Hanning window. Power spectra for the adjusted velocities of the AF method, utilized in the discussion, were estimated in a similar manner. For the wave-free Elkhorn M1 and Elkhorn M12 datasets, an artificial wave band cutoff frequency of $0.1 \mathrm{~s}^{-1}$ was set to estimate CF method stresses under conditions similar to those found in the other datasets.

\section{Results}

\section{a. Analysis of method applicability and uncertainties}

The CF method criteria described above provide guidance regarding when the method might give viable results as well as when the results should be trusted. Of the wavecontaining datasets used here, the wave/drift criterion was met a maximum of $60 \%, 20 \%$, and $70 \%$ of the time for Santa Barbara, Moorea, and MVCO, respectively (Fig. 3). Additionally, the CF method results were within the wavenumber bounds in approximately $50 \%, 70 \%$, and $70 \%$ of observations, respectively. The CF method stress estimates satisfied both criteria in a maximum of
$40 \%, 10 \%$, and $45 \%$ of the available bursts (Fig. 3 ). The wave/drift threshold generally dominates the combined metric and, from this criterion alone, it is evident that application of the CF method in conditions similar to those at Moorea is not worthwhile.

Following the differences seen in the wave climates (Fig. 1), significant differences of the wave band cutoff frequency (or period) exist between the sites. Cutoff periods at Moorea were quite long, approaching $20 \mathrm{~s}$ narrowbanded. At Santa Barbara, the cutoff periods were wider in bandwidth but similar at all depths (Fig. 1). In contrast, cutoff periods at MVCO were both wide-banded in frequency/period and varied significantly over the water column.

The uncertainties in stress estimates varied significantly between deployments because of differences in flow conditions and sampling strategies, and within deployments when flow conditions varied. For each stress estimate, the uncertainties were bin-averaged by the estimated stresses and, for simplicity, the zero-stress bin average is shown in Table 1 to allow comparison among the datasets and methods. As stress uncertainties generally increase with stress magnitude as well as wave conditions, these zero-stress uncertainties should be thought of as the minimum error values of the method or deployment. However, as no correlation factor is used here for the AF error results, the true level for these methods is likely to be 2 or more times higher (Williams and Simpson 2004).

Regardless, the levels shown in Table 1 serve as a useful comparison of the stress uncertainty between methods and between the mode 1 and mode 12 sampling strategies. For the Santa Barbara data, uncertainties using the AF method were a factor of 4 less than those using the $\mathrm{CF}$ method, and similar in both beam axes. In contrast, the mode $1 \mathrm{MVCO}$ data has baseline uncertainties of $0.045 \mathrm{~Pa}$ for the AF method and 0.09 Pa for the CF methodperhaps comparable if the autocorrelation correction factor was applied. For Moorea, only the AF results, strongly dependent on the flow direction, are reported here as the CF method could not be used for most of the dataset. For the two nonwave datasets, baseline uncertainties of the basic variance method are also included (Table 1). These are generally similar to, or slightly lower than, the AF method uncertainties. CF method uncertainties were similar to VM uncertainties in Elkhorn M1, the mode 1 dataset, where the smaller $0.25-\mathrm{m}$ bin thicknesses led to vastly increased errors compared to MVCO results. CF method uncertainties were 3-4 times greater than VM and $\mathrm{AF}$ uncertainties for the Elkhorn M12 dataset. VM and AF uncertainties using the mode 12 instrument were up to an order of magnitude less than those found using the mode-1 instrument. 

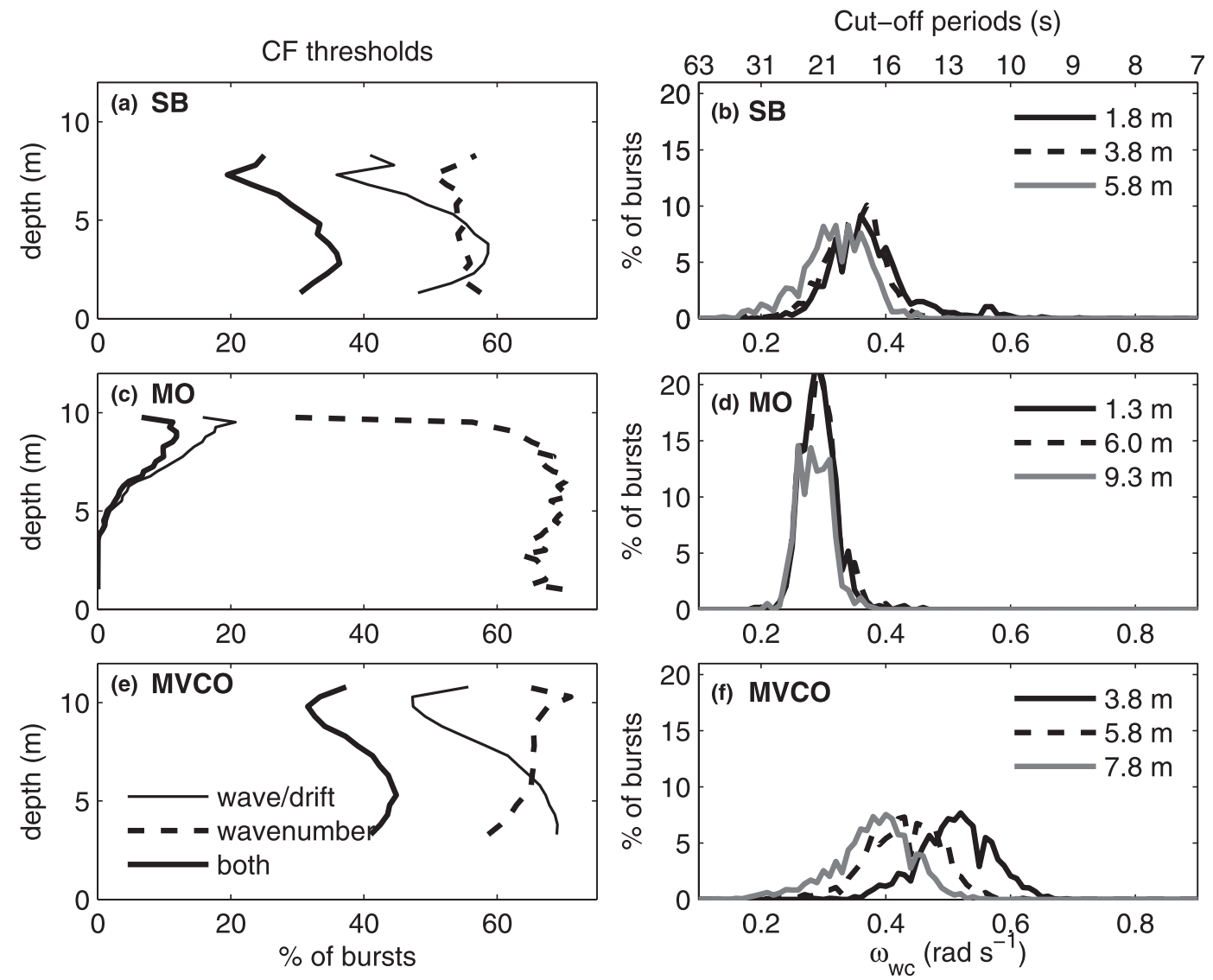

FIG. 3. Criteria for determining if and where the CF method can be applied for (a),(b) Santa Barbara; (c),(d) Moorea; and (e),(f) MVCO. Panels (a),(c), and (e) show the percent of bursts when the wave/drift velocity ratio was less than 2 (thin solid line), the modeled roll-off wavenumber was less than the cutoff wavenumber (dashed line), and when both occurred (thick solid line). Panels (b),(d), and (f) show histograms of the cutoff frequency (or period), defined as when a pressure-based vertical velocity spectra exceeds $30 \%$ of the average beam velocity spectra, for three different depths.

\section{b. Comparisons between near-bottom stress estimates and quadratic drag}

Estimated stresses in the bottom-most velocity bin were compared with estimates of the quadratic drag law $(\mathbf{U}|\mathbf{U}|)$, calculated from the burst-mean horizontal beam 1-2 and 3-4 axis velocities to evaluate performance differences among the techniques (Figs. 4, 5; Table 2). However, the CF method performance criteria exclude the use of the CF method at Moorea throughout the water column during a large fraction of the deployment (Fig. 3). Given the instrument's position in a channel on the reef, the velocities and stresses estimated at 6 meters above bottom (m.a.b.) are used here, following Rosman et al. (2008). Note that all correlations and regressions are shown in the figures, while only those results that were significantly different from zero, at a $95 \%$ confidence level found using the effective degrees of freedom (Chelton
1983), are included in the summary given in Table 2. Uncertainty estimates for the drag coefficients are based on the $95 \%$ confidence interval of the linear fit to the measurements and do not account for biases in stress estimates. For example, consistent under or overprediction of stresses would result in a corresponding under- or overestimation of $\mathrm{C}_{D}$ not accounted for in the uncertainties reported in Table 2.

Comparisons between near-bottom stress and the quadratic drag law reveal distinct variations in method performance among the datasets containing waves. For the conditions present near the bottom at Santa Barbara, correlations and regressions with the quadratic drag law for the AF and CF stress estimates agree within uncertainty limits. Both methods give $r^{2}$ values near 0.3 and 0.55 for beams $1-2$ and $3-4$ and realistic drag coefficients (1.5 and $2 \times 10^{-3}$ ) for beams 1-2 and 3-4 (Fig. 4; Table 2). At 6 m.a.b. at Moorea, correlations between CF stress 

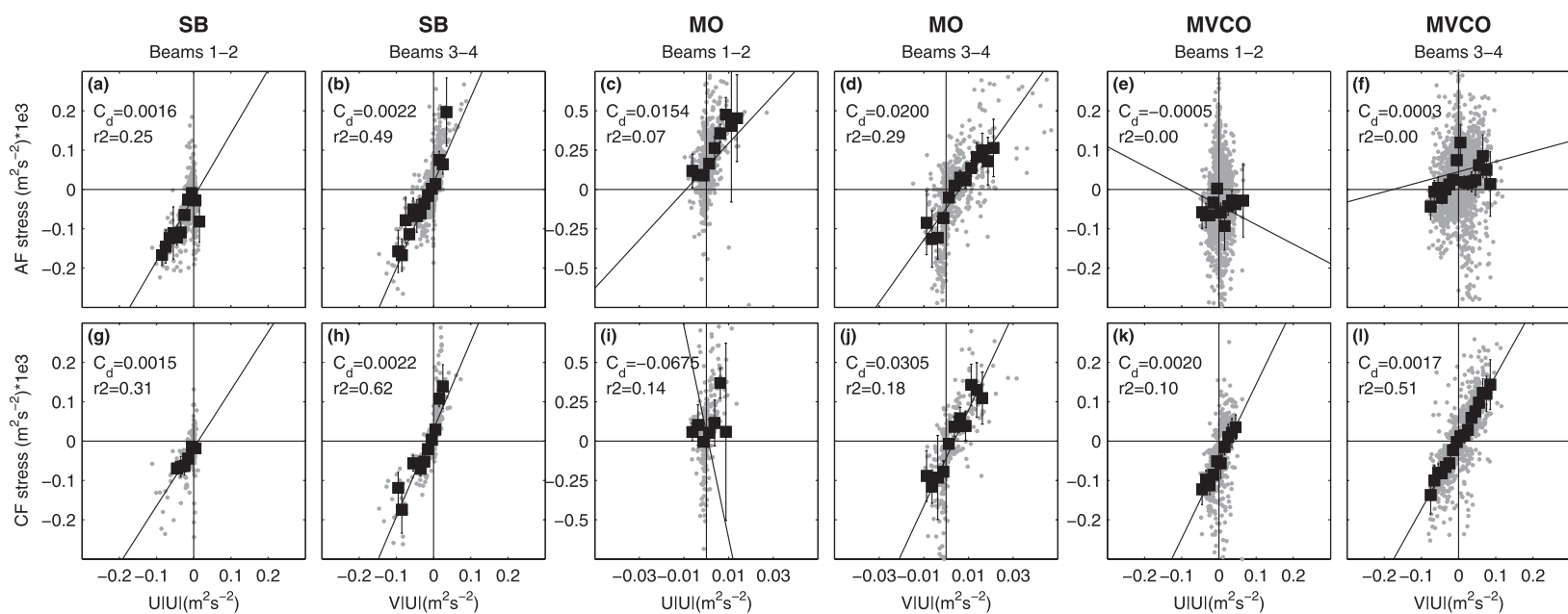

FIG. 4. Scatterplot (gray) and bin-averaged (black squares) comparisons of (a)-(f) AF and (g)-(l) CF method Reynolds stresses to a quadratic drag law for Santa Barbara [in (a),(b),(g), and (h)], Moorea [in (c),(d),(i), and (j)] and MVCO [in (e),(f),(k), and (l)]. The quadratic drag law was calculated using burst-mean velocities from the bottommost ADCP bin for Santa Barbara and MVCO, but a bin $6 \mathrm{~m}$ above the bottom for Moorea, following Rosman et al. (2008). Linear regressions, slopes $\left(C_{d}\right)$, and correlation coefficients $\left(r^{2}\right)$ between the raw (not bin averaged) time series are shown in each panel. For each bin-averaged stress, binned by the values on the $x$ axis, standard error bounds were estimated using the effective degrees of freedom (Chelton 1983).

estimates and the quadratic drag law were not significant along both axes. Here, AF method correlations were not significant for beams 1-2, but significant at $r^{2}=0.29$ for beams 3-4. The AF method beam 3-4 drag coefficient was $2.0 \times 10^{-2}$. For the conditions at MVCO during January 2008, correlations between the AF method stress and the quadratic drag law were not significant (Fig. 4; Table 2). In contrast, CF method stress correlations with the quadratic drag law were significant for both directions with $r^{2}$ values up to 0.51 . Drag coefficients estimated using the CF method at MVCO were consistent with previous estimates in the region (J. Trowbridge 2008, personal communication).

For the wave-free Elkhorn datasets, bottom stress comparisons were made using stress estimates for $\mathrm{CF}, \mathrm{AF}$, and VM (Fig. 5; Table 2). For the 1-2 direction of the Elkhorn M1 dataset, stresses calculated using all methods had significant scatter about small variations in $U|\mathbf{U}|$. In the 3-4 direction, Elkhorn M1 estimated stress was large with similar correlations $\left(r^{2}=0.72\right)$ for all methods. The
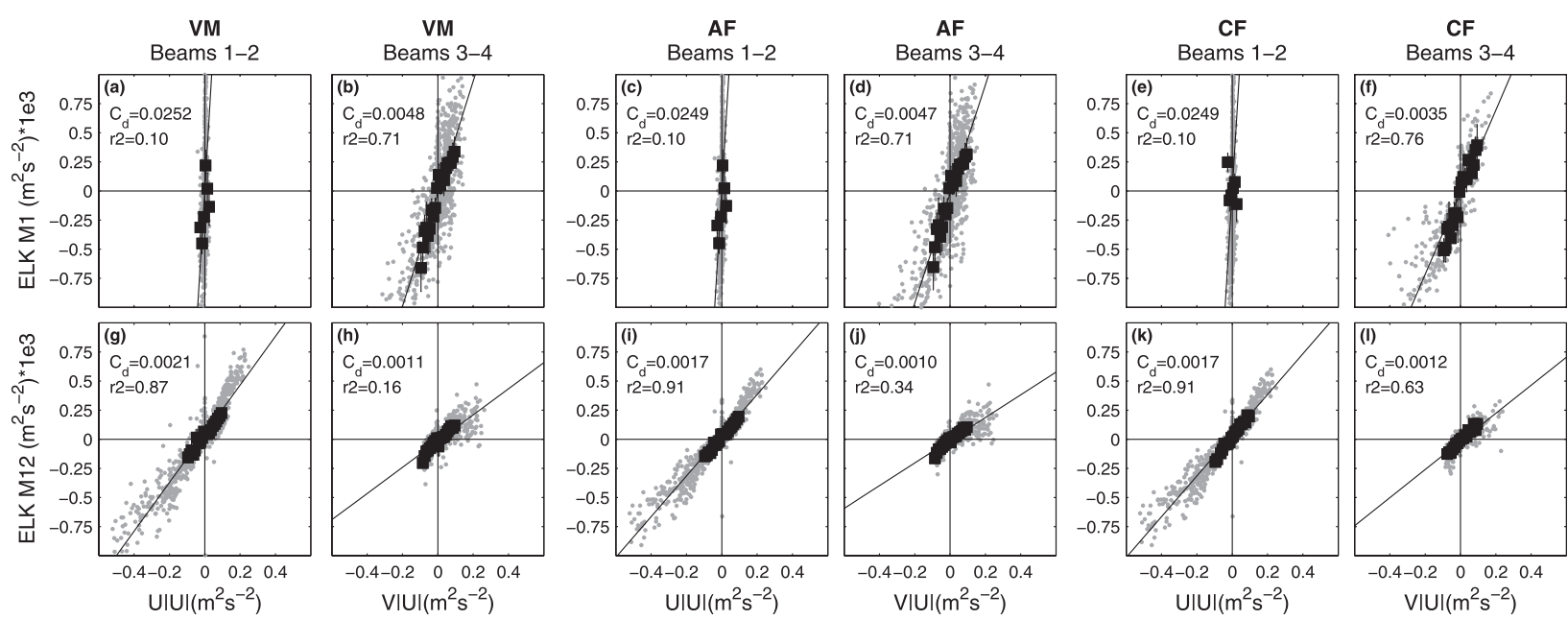

FIG. 5. Scatterplot (gray) and bin-averaged (black squares) comparisons of (a),(b),(g),(h) VM; (c),(d),(i),(j) AF; and (e),(f),(k),(l) CF method Reynolds stresses to a quadratic drag law for the Elkhorn M1 [in (a)-(f)] and Elkhorn M12 [in (g)-(l)] datasets. For both instruments, the quadratic drag law was calculated using burst-mean velocities from the bottommost ADCP bin. Bin averages and linear regressions are calculated as described in Fig. 4. 
TABLE 2. Correlations and regressions between measured stress and a quadratic drag law. Nonsignificant correlations are shown in italics. Regressions are listed for significant correlations only.

\begin{tabular}{|c|c|c|c|c|c|c|c|c|c|c|c|c|}
\hline & \multicolumn{6}{|c|}{ Beams 1-2 } & \multicolumn{6}{|c|}{ Beams 3-4 } \\
\hline & \multicolumn{2}{|c|}{$\mathrm{VM}$} & \multicolumn{2}{|c|}{$\mathrm{AF}$} & \multicolumn{2}{|r|}{$\mathrm{CF}$} & \multicolumn{2}{|c|}{ VM } & \multicolumn{2}{|r|}{$\mathrm{AF}$} & \multicolumn{2}{|r|}{$\mathrm{CF}$} \\
\hline & $r^{2}$ & $C_{d}^{*}$ & $r^{2}$ & $C_{d}^{*}$ & $r^{2}$ & $C_{d}^{*}$ & $r^{2}$ & $C_{d}^{*}$ & $r^{2}$ & $C_{d}^{*}$ & $r^{2}$ & $C_{d}^{*}$ \\
\hline Santa Barbara & & & 0.25 & $1.6 \pm 0.2$ & 0.31 & $1.5 \pm 0.3$ & & & 0.49 & $2.2 \pm 0.1$ & 0.62 & $2.2 \pm 0.2$ \\
\hline Moorea & & & 0.07 & & 0.14 & & & & 0.29 & $20.0 \pm 2.3$ & 0.18 & \\
\hline MVCO & & & 0.0 & & 0.10 & $2.0 \pm 0.5$ & & & 0.0 & & 0.51 & $1.7 \pm 0.1$ \\
\hline Elkhorn M1 & 0.10 & & 0.10 & & 0.10 & & 0.71 & $4.8 \pm 0.2$ & 0.71 & $4.7 \pm 0.2$ & 0.76 & $3.5 \pm 0.2$ \\
\hline Elkhorn M12 & 0.87 & $2.1 \pm 0.1$ & 0.90 & $2.0 \pm 0.1$ & 0.90 & $2.0 \pm 0.1$ & 0.16 & & 0.42 & $1.1 \pm 0.1$ & 0.63 & $1.2 \pm 0.1$ \\
\hline
\end{tabular}

$* \times 10^{-3}$.

drag coefficient for the CF method $\left(3.5 \times 10^{-3}\right)$ was noticeably different than that for AF and VM (both near $\left.4.7 \times 10^{-3}\right)$ and agreed with an independent estimate $\left(3.5 \times 10^{-3}\right)$ based on collocated ADV observations from the same experiment (N. Nidzieko 2011, personal communication) For the Elkhorn M12 dataset, beams 1-2 were approximately $30^{\circ}$ from the along-channel direction. Along the 1-2 axis, $\mathrm{CF}, \mathrm{AF}$, and $\mathrm{VM}$ have similar correlations (0.9) and drag coefficients $\left(1.7\right.$ to $\left.2.1 \times 10^{-3}\right)$, agreeing well with ADV-based along-channel estimates (N. Nidzieko 2011, personal communication). In the 3-4 direction, drag coefficients were very similar for all three methods but correlations varied substantially $\left(r^{2}=0.16\right.$, 0.34 , and 0.63 for VM, AF, and CF, respectively).

Additional stress comparisons under varying levels of observed significant wave height were made by expanding the MVCO dataset considered to the full two winters of observations available. The longer record length allows statistically significant, near-bottom stress comparisons to be made for varying significant wave heights. Shown in Fig. 6 for the dominant 3-4 beam axis only, correlation $\left(r^{2}\right)$ values for the CF method are generally constant at 0.5 for much of the range considered. Variance methodbased $\left(r^{2}\right)$ values give drag coefficients that are similar to $\mathrm{CF}$ method results below significant wave heights of $1 \mathrm{~m}$. In contrast, the AF method $r^{2}$ values decreased from near 0.2 to 0 as wave heights increase from 0.4 to $2 \mathrm{~m}$. Drag coefficients for the CF method increase with increasing wave height, from 1.4 to $2 \times 10^{-3}$ over the same wave height range. Such an increase with wave height is consistent with Grant and Madson's (1979) theory of wave contributions on bottom stress.

\section{c. Comparisons between near-surface stress estimates and wind stress}

Because of the availability of quality wind observations at the nearby tower, near-surface stress estimates for the MVCO dataset using both the CF and AF methods were compared to the estimated wind stress using hourly averaged stress estimates (Fig. 7). In the surface-most bin, at $1.5 \mathrm{~m}$ below the surface, CF method stress was positively correlated with the ASIT wind stress, having $r^{2}$ values of 0.61 and 0.38 for the $1-2$ and 3-4 axes, respectively. Regression coefficients between the wind stress and ADCP-based stress at this depth were 0.91 and 0.87 for the 1-2 and 3-4 axes, respectively. In contrast, at the surface-most stress measurement of the AF method, $4.0 \mathrm{~m}$, the wind stress comparisons had $r^{2}$ values of 0.20 and 0.05 for the 1-2 and 3-4 axes, indicating no relationship. CF method comparisons at $4 \mathrm{~m}$ below the surface had higher correlations and larger slopes $(0.65-0.70$; not shown here) than the AF results shown here.

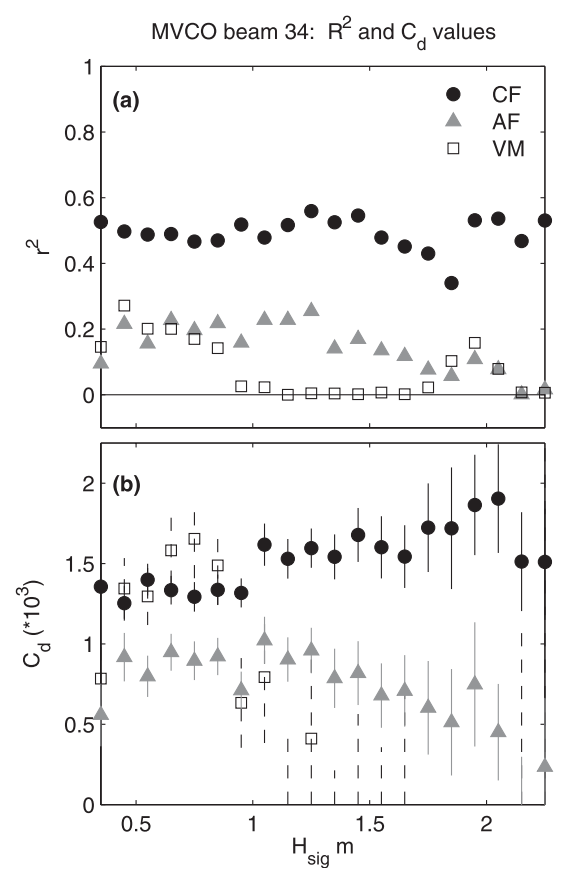

FIG. 6. Comparison of the (a) correlations (as $r^{2}$ values) and (b) drag coefficients between $\mathrm{AF}, \mathrm{CF}$, and VM bottom stress against a quadratic drag law for all 2008 and 2009 wintertime (1 Oct-31 Mar) data for the 3-4 beam axis of the MVCO dataset. 
MVco

Beams 1-2
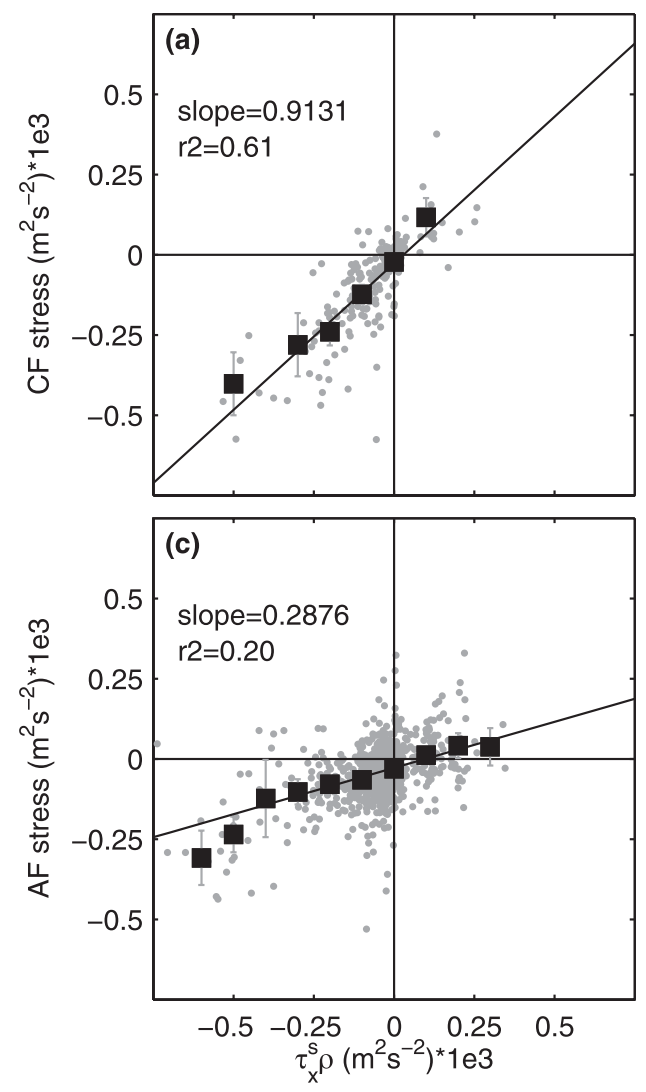

MVCO

Beams 3-4
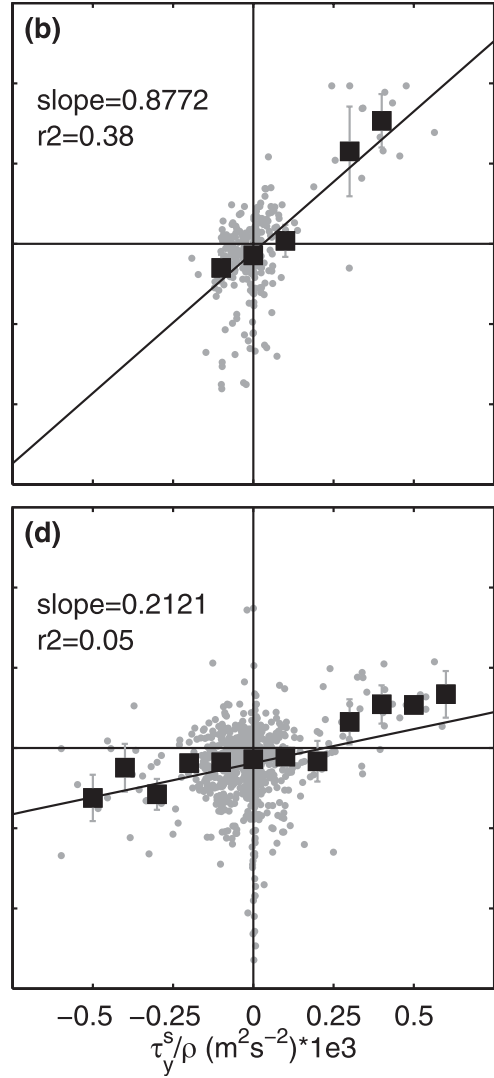

FIG. 7. Scatterplot (gray) and bin-averaged (black squares) comparisons of near-surface (a),(b) CF (at 1.5-m depth below surface) and (c),(d) AF (at 4-m depth below surface) method Reynolds stresses to nearby estimates of the surface wind stress for MVCO. Bin averages and linear regressions are calculated as described in Fig. 4.

\section{d. Comparisons of the vertical structure of stress}

The full winter dataset at MVCO was also used to examine the estimated vertical structure of Reynolds stresses. Hourly averages of velocities, winds, and estimated stress vectors were rotated into an along- and across-shore coordinate system defined for MVCO following Lentz et al. (2008). The mean stress responses to particular types of wind forcing events were isolated to compare the performance-in terms of potential residual biases and differences in vertical coverage-of the $\mathrm{AF}$ and $\mathrm{CF}$ methods at MVCO. Although all types of wind events were examined, the average results for times of alongshore (eastward) wind stress between 0.035 and $0.125 \mathrm{~Pa}$, having a mean of $0.06 \mathrm{~Pa}$, are shown in Fig. 8 .

The mean structure of the alongshore and acrossshore velocity for these conditions is vertically sheared with stronger alongshore (eastward) and offshore flow at the surface, and decaying with depth. Note that the depth-mean across-shore velocities have not been subtracted from the observed mean vertical profile, as is typically done to isolate the wind-forced across-shore exchange. Comparing vertical structure of stress using the $\mathrm{AF}$ and $\mathrm{CF}$ methods during these upwelling-favorable wind conditions, the CF method is able to span a greater fraction of the water column than the AF method, and matches the surface wind stress in the near-surface bin for the downwind direction. Deeper in the water column, from 3 to 7 m.a.b., the two might have a similar trend in the alongshore direction with a maximum stress around 4.5 m.a.b., but the AF stresses are weaker than the CF stresses. The across-shore stress is near zero at the surface for the CF method but becomes increasingly positive with depth, a trend only somewhat echoed by the AF method stress. The differences between the estimated mean stresses for each of the methods shown here are representative other wind directions and magnitudes. 

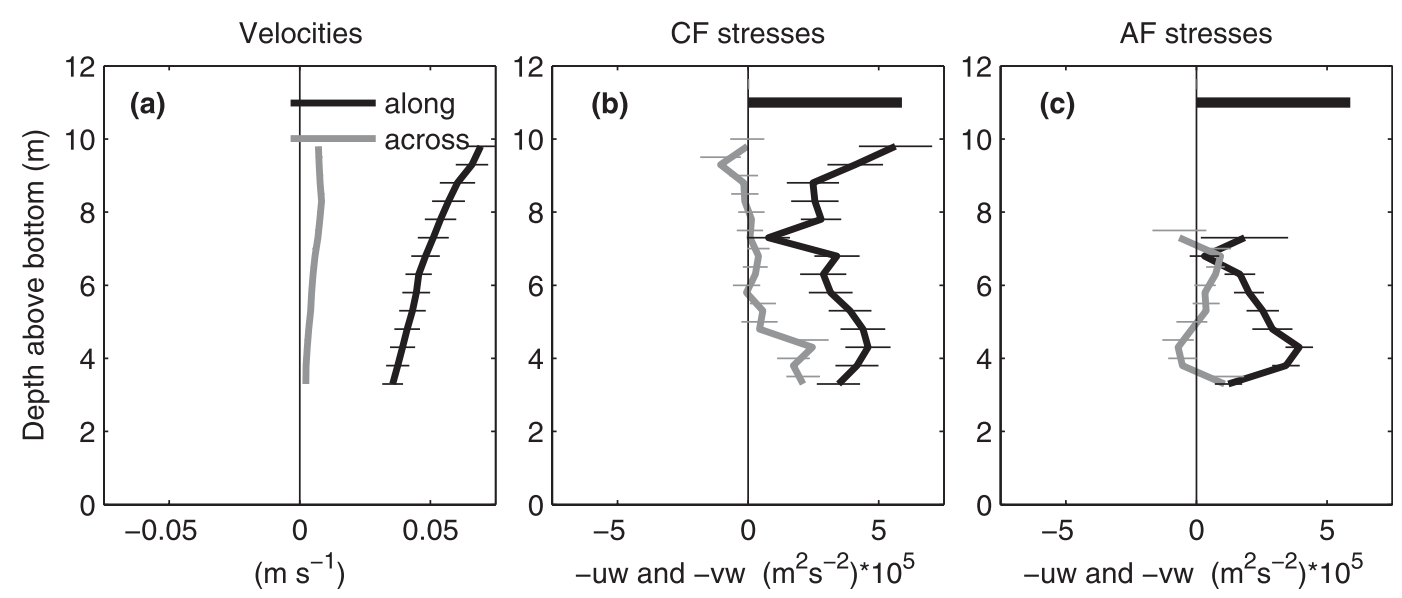

FIG. 8. MVCO mean response of (a) velocity, (b) CF method stress, and (c) AF method stress to moderate alongshelf wind stresses (mean of $0.06 \mathrm{~Pa}$ ) when both surface gravity waves and across-shelf winds were small. In each panel the along-shelf velocity or stress is shown in thick black while the across-shelf is in thick gray, both with standard error bars for each bin. Thick horizontal lines at 11-m height above bottom in the stress panels represent the measured wind stress. Of the full two winters used in the extended MVCO dataset, 601 hourly observations with valid CF observations matched the wave and wind criteria described above.

\section{e. Cospectral view of method performance}

A more detailed look at the performance of the methods at all sites except Moorea can be made by comparing the observed frequency structure of the turbulent cospectra directly to the Kaimal et al. (1972) model. These comparisons allow an assessment of both the representativeness of the theoretical model used in obtaining the $\mathrm{CF}$ method stress, and the potential differences in the observed cospectra for each of the methods. To collapse the results from each method at Elkhorn M12 and Elkhorn M1, as well as for small and large wave conditions at both Santa Barbara and MVCO, the integrated cospectra-the ogive curves-from all bursts in the bottommost velocity bin were normalized by $\mathrm{CF}$ method estimated roll-off wavenumber, as $\log 10\left(k / k_{o}\right)$, and by the CF method estimated stress. The median ogive curves for bins of normalized wavenumber, with bootstrapped standard errors, are shown in Fig. 9.

For both Elkhorn M12 and Elkhorn M1, normalized ogive curves were similar for the variance method (i.e., the uncorrected cospectra), the AF method, and the $\mathrm{CF}$ method (i.e., the below-waveband portion of the uncorrected cospectra). As shown in Figs. 9a,d, all methods fall close to the theoretical line for wavenumber ratios less than 0.75, where the VM and AF ogive curves climb above the theoretical curve while the $\mathrm{CF}$ curves fall on or slightly below it. Use of AF method bin separations less than $3 \mathrm{~m}$ (not shown here) had the effect of reducing the energy in the AF method ogive curves at and above the roll-off wavenumber. This indicates that AF method results with separations less than $3 \mathrm{~m}$ were eliminating some of the turbulent fluctuations present along with wave velocities.

For the Santa Barbara and MVCO datasets, velocity bursts were divided into two groups based on the significant wave height present: small waves, defined here as $0.5 \mathrm{~m} \leq H_{\text {sig }}<0.75 \mathrm{~m}$, and large waves, defined here as $0.75 \mathrm{~m} \leq H_{\text {sig }}<1.5 \mathrm{~m}$. These ranges were chosen such that in both, the mean significant wave heights were similar at both Santa Barbara and MVCO. For the Santa Barbara dataset, both the AF and $\mathrm{CF}$ method ogive curves fall close to the theoretical model curve for all but the highest wavenumbers (Figs. 9b,e). The differences seen at $\log 10\left(k / k_{o}\right)=0.5$ for the AF method during small waves, and at $\log 10\left(k / k_{o}\right) \geq 1$ for both methods during both types of conditions are close to, or within, the bootstrapped standard error bounds estimated. In contrast to Santa Barbara, the mean AF method ogive curves for MVCO deviate from the model and CF methods for both small and large wave conditions near $\log 10\left(k / k_{o}\right) \geq$ 0 , attaining slightly smaller values than the theory for higher wavenumbers (Figs. 9c,f). These deviations are unrelated to the separation distance used in the $\mathrm{AF}$ method, as was seen with Elkhorn M12 and M1 datasets. The AF curves underestimate the model for both larger and smaller separations.

\section{Discussion}

The results shown here indicated that all three methods-VM, AF, and $\mathrm{CF}$ - can provide reasonable estimates of Reynolds stresses under certain conditions. However, the performance of the methods varies with 

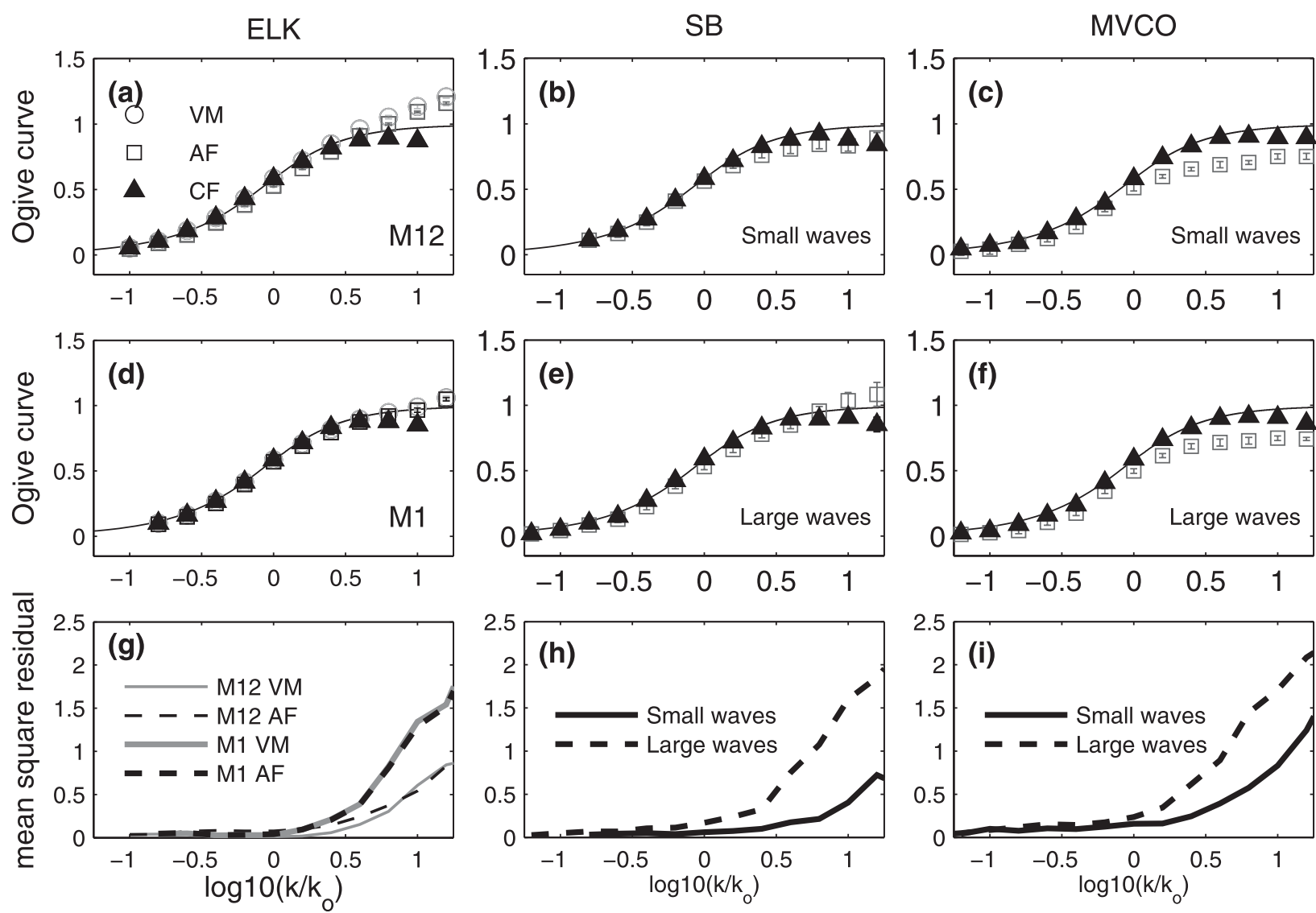

FIG. 9. Comparison between measured ogive curves and the Kaimal et al. (1972) theoretical model. (a),(d) Bin medians for the Elkhorn M12 and Elkhorn M1 VM, AF, and CF methods, normalized by the roll-off wavenumber, $k_{o}$, and stress estimated using the CF method. The theoretical ogive curve is shown as the solid line. Similar bin medians are shown for both small $\left(0.5 \mathrm{~m} \leq H_{\text {sig }}<0.75 \mathrm{~m}\right)$ and large $\left(0.75 \mathrm{~m} \leq H_{\text {sig }}<1.5 \mathrm{~m}\right.$ ) wave conditions at (b),(e) Santa Barbara and (c),(f) MVCO. These small and large wave ranges were chosen such that Santa Barbara and MVCO have similar significant wave heights and periods in both ranges. (g),(h),(i) The mean-square residual between the AF [and VM for (g)] and the Kaimal et al. (1972) model for both Elkhorn datasets (g), Santa Barbara (h), and MVCO (i).

both wave climate and the sampling characteristics of the instrument, most specifically mode 1 versus mode 12 . These two factors are discussed below to explain the variable performance of the methods on the five datasets used in this manuscript.

\section{a. The role of wave climate}

The occurrence of variable dominant wave periods translates into differences in the wave band cutoff frequencies $\left(\omega_{\mathrm{wc}}\right)$ used in the CF method for each dataset, and in part the performance of the method. At MVCO, the cutoff frequency decreased as the surface is approached, while at both Santa Barbara and Moorea, wave band cutoffs are lower in frequency (longer period) and mostly uniform with depth (Fig. 3). When the dominant surface gravity waves have long periods and the wave band cutoff is lower in frequency, the CF model fit is made over a smaller range of frequencies. Fits made over a smaller spectral domain are more likely to result in 1) an estimated roll-off wavenumber that is higher than the wave band cutoff; 2) a poor fit, as judged by the percent variance explained, since most of the model cospectra's structure is around the roll-off wavenumber; or 3) both. For quality control purposes, fits meeting any of these conditions were excluded from the analysis. The first criterion was exceeded most often at Santa Barbara, where up to $50 \%$ of bursts considered had estimated rolloff wavenumbers that exceeded the wave band cutoff (Fig. 3). Given that wave band cutoff frequencies at Santa Barbara were equal to or greater than those at Moorea, it appears more likely that the differences in performance were driven by higher roll-off wavenumbers (shorter length scales) at Santa Barbara. Stratification, which reduces the dominant turbulent length scales and thereby increases the roll-off wavenumber, has a similar effect.

The quality of the AF method results can be significantly affected by the wave climate. For narrow wave 
spectra, the signal-to-noise ratio is higher, thus giving better estimates of the wave velocities present. Additionally, the AF method also performs better with lower-frequency waves which have wave orbital velocities that are more uniform with depth. For shorter period waves having orbital velocities with more significant vertical structure, the predictions at one bin-based on measurements at another-can be less accurate.

For the CF method, it is not just the wave climate but the combination of waves and currents that control when viable results are possible. The threshold ratio of the wave velocity over drift velocity serves as a more useful metric of whether the CF method is appropriate than the wave climate itself and can be estimated a priori. The differences between this ratio for Santa Barbara and Moorea, having somewhat similar wave periods, are strongly controlled by the currents present (Fig. 3). While conditions at Santa Barbara were below the threshold value of 2 more than $50 \%$ of the time, for conditions such as those at Moorea-weak flows at Moorea and strong swell-the threshold was surpassed in up to $80 \%$ of bursts, and thus the CF method is essentially unable to obtain unbiased results. The AF method lacks similar a priori estimates of method performance.

\section{$b$. The role of instrument noise}

Comparisons between the wave-free stress estimates at Elkhorn Slough and a quadratic drag law are generally similar for the three methods (Fig. 5). However, the drag coefficient estimated using the CF method stress agrees more closely with independent estimates of the drag coefficient when the noisier mode 1 sampling is used. This difference is likely due to the fact that the CF method ignores high-frequency fluctuations for which noise is a significant fraction of the signal. However, where the signal-to-noise ratio falls or low-frequency errors exist in the stress estimates as well (i.e., beams 1-2 for Elkhorn M1), this advantage is not helpful.

The contribution of high-frequency noise to individual stress estimates is averaged out in the bin-median ogive curves; for example, VM and AF ogive curves are similar for Elkhorn M1 and M12 (Figs. 9a,d). However, the effect of high-frequency noise can be seen in the rootmean-square of the differences (rms residuals) between the AF and VM ogive curves and the model (Fig. 9g). Using this quantity, the effect of noise appears as a nonzero normalized rms residual. For both M12 and M1, rms residuals increase with wavenumber for $\log 10\left(k / k_{o}\right)>0.5$ with little difference between either the VM or AF results. However, the rate of increase and total variance is about twice as large for Elkhorn M1 than for Elkhorn M12. It is likely that this noise-related effect acts to reduce the effectiveness of the AF method, relative to the
CF method, when the mode 1 sampling is being used, as is the case at MVCO.

\section{c. Contrasting Santa Barbara and MVCO}

These two factors combined (wave climate and instrument noise) offer an explanation for why the AF and $\mathrm{CF}$ methods give similar results for Santa Barbara, but quite different results for MVCO. The wave heights at MVCO were larger and wave frequencies were higher and more broadbanded than at Santa Barbara. Additionally, mode 1 sampling was used at MVCO as opposed to mode 12 at Santa Barbara, thus AF method results at MVCO were noisier while the CF method omits the higher frequencies where noise would dominate. Both of these factors would tend to reduce the performance of the AF method and hence increase the benefits of the CF method. For these reasons, the AF method might have had problems removing the wave bias and keeping the turbulent energy for MVCO, but was more effective for Santa Barbara. Additionally, variations in the optimal separation distance for the AF method might act to decrease the mean ogive curve relative to the theoretical curves at moderate to high frequencies as it would, on average, remove turbulent energy in addition to the waves. Such decreases are observed in the AF method mean ogive curves for MVCO (Figs. 9c,f).

The combined effect of noise and waves on the performance of the AF method can be quantified by considering the means and rms residuals of the AF ogive curves from the theoretical model at Santa Barbara and MVCO (Figs. 9h,i). While the mean difference captures the bias present in the stress estimate relative to the model, rms residuals capture both the bias and the level of noise present. For the Santa Barbara dataset during small waves, the residual increased slowly to 0.4 (normalized units) at $\log 10\left(k / k_{o}\right)=1$, and then more rapidly to near 0.75 at $\log 10\left(k / k_{o}\right)=1.2$ (Fig. 9h). With larger waves, the rms residual at Santa Barbara was larger at 0.5 at $\log 10\left(k / k_{o}\right)=0.5$ but reached 1.5 at $\log 10\left(k / k_{o}\right)=$ 1. At MVCO during small wave conditions, the residual increased rapidly with normalized wavenumber to 0.75 at $\log 10\left(k / k_{o}\right)=1$ and 1.3 at $\log 10\left(k / k_{o}\right)=1.2$ (Fig. 9i). During large waves at MVCO, the residual was larger still, reaching 0.75 at $\log 10\left(k / k_{o}\right)=0.5$ and 1.75 at $\log 10\left(k / k_{o}\right)=1$. Thus, both Santa Barbara and MVCO have increases in the mean-square residual between the AF method cospectra and the theoretical model as the significant wave height increases. Yet, both the small and large wave residuals are larger at MVCO compared to Santa Barbara. The difference between the Santa Barbara and MVCO rms residuals is similar for both large and small wave conditions, approximately $0.25-0.4$. Thus, this difference is assumed to be the "noise" component of 
the residual or the part of the total errors due just to the changes in instrument sampling methods.

Coupling these results with the mean ogive curve comparisons shown earlier, the differences between Santa Barbara and MVCO results for the AF method are twofold. First, for similar wave heights, an overall bias of the stress exists along with increased rms residuals at higher wavenumbers. Both appear related to the increased noise present in the MVCO dataset, presumably because the instrument used mode 1 sampling. Second, wave heights at MVCO were larger than at Santa Barbara (Fig. 1) and, as shown by the rms residuals in Fig. 9, larger wave heights lead to larger deviations of individual AF method results from theory. Thus, both differences in noise levels as well as differences in wave climate contributed to the poor performance of the AF method at MVCO compared with Santa Barbara.

\section{Summary}

This study compared the performance of a number of methods for estimating Reynolds stresses from ADCP measurements in the presence of waves (the variance method, the adaptive filtering method, and the cospectral fitting method) for five datasets covering a wide range of wave and current conditions in estuaries and the coastal ocean. Based on the analyses of these data, the standard variance method performs as well as the $\mathrm{AF}$ and $\mathrm{CF}$ methods if there are no waves and noise in the raw measurements is small. The AF method performs well if waves are low frequency and/or narrow-banded and the noise in raw measurements is small. If waves are broadbanded and high frequency, the AF method performs poorly, in part because predictions of wave velocities at one bin-based on measurements at another-are less accurate. Thus, the CF method is more appropriate than the AF method for broad-banded and high-frequency waves. The AF method also cannot be used to estimate stresses very close to the surface because of the separation distance needed between the depth of interest and the depth used to form the filter weights. Thus, the CF method can often be used over a greater portion of the water column. Performance using the AF method degrades as the noise in raw measurements increases, particularly at higher frequencies; thus, the CF method is generally the preferred method if measurements were collected in mode 1 .

However, the CF method is restricted to wave orbital velocities that are similar in magnitude to currents because the method assumes a particular shape for the turbulence cospectrum. If wave orbital velocities are large relative to current speeds, turbulent energy is aliased from low frequencies into the wave band, altering the shape of the turbulence cospectrum. In general, if $u_{w \mathrm{rms}} / U_{d}>2$ often, then the $\mathrm{CF}$ method should not be used, and the AF method is preferred. Also, the CF method cannot be used if wave periods are long relative to the roll-off frequency, the frequency corresponding to advection of dominant turbulent eddies past the sensor by the current, because there is not sufficient frequency range below the wave peak to accurately fit the model cospectrum. Thus, if wave periods are long or the dominant turbulent eddies are small (e.g., because of stratification), the AF method is more appropriate.

In summary, the choice of method will depend primarily on wave conditions (wave periods, spectral width, ratio of wave orbital velocity to current), and measurement noise (e.g., mode 1 versus mode 12). It is useful to estimate these quantities when designing a deployment to evaluate whether stresses can be estimated from ADCP measurements with sufficient accuracy. In general, it is best to use the instrument configuration that will achieve the highest accuracy measurements. Here we have compared mode 1 and mode 12, but pulse coherent modes (mode 11) can further improve measurement accuracy, although they are presently limited to relatively small vertical ranges and small instantaneous velocities. Regardless of the method used, uncertainties in stress estimates from ADCPs are much larger than point measurements from ADVs, and these uncertainties increase in wavy conditions. It is therefore critical to be mindful of uncertainties when using stress estimates derived from ADCP measurements.

Acknowledgments. The authors thank Jim Hench, Brian Gaylord, Janet Fredericks, and Nick Nidzieko for their efforts in collecting and providing the Moorea, Santa Barbara, MVCO, and Elkhorn Slough datasets used in this study. J. Rosman acknowledges funding from the National Science Foundation (OCE-1061108). This manuscript was improved by the helpful comments of three reviewers.

\section{REFERENCES}

Chelton, D., 1983: Effects of sampling errors in statistical estimation. Deep-Sea Res., 30, 1083-1101.

Gerbi, G., J. Trowbridge, J. Edson, A. Plueddemann, E. Terray, and J. Fredericks, 2008: Measurements of momentum and heat transfer across the air-sea interface. J. Phys. Oceanogr., 38, 1054-1072.

Grant, W., and O. Madson, 1979: Combined wave and current interaction with a rough bottom. J. Geophys. Res., 84, 17971808.

Kaimal, J., J. Wyngaard, Y. Izumi, and O. Cote, 1972: Spectral characteristics of surface-layer turbulence. Quart. J. Roy. Meteor. Soc., 98, 563-589. 
Kirincich, A., S. Lentz, and G. Gerbi, 2010: Calculating Reynolds stresses from ADCP measurements in the presence of surface gravity waves using the cospectra fit method. J. Atmos. Oceanic Technol., 27, 889-907.

Large, W., and S. Pond, 1981: Open ocean momentum flux measurements in moderate to strong winds. J. Phys. Oceanogr., 11, 324-336.

Lentz, S., M. Fewings, P. Howd, J. Fredericks, and K. Hathaway, 2008: Observations and a model of undertow over the inner continental shelf. J. Phys. Oceanogr., 38, 2341-2357.

Lohrmann, A., B. Hackett, and L. Roed, 1990: High-resolution measurements of turbulence, velocity, and stress using a pulseto-pulse coherent sonar. J. Atmos. Oceanic Technol., 7, 19-37.

Lu, Y., and R. Lueck, 1999: Using a broadband ADCP in a tidal channel. Part II: Turbulence. J. Atmos. Oceanic Technol., 16 , 1568-1579.

Lumley, J., and E. Terray, 1983: Kinematics of turbulence convected by a random wave field. J. Phys. Oceanogr., 13, 2000-2007.

Nidzieko, N., D. Fong, and J. Hench, 2006: Comparison of Reynolds stress estimates derived from standard and fast-ping ADCPs. J. Atmos. Oceanic Technol., 23, 854-861.

Rippeth, T., E. Williams, and J. Simpson, 2002: Reynolds stress and turbulent energy production in a tidal channel. J. Phys. Oceanogr., 32, 1242-1251.

_- J. Simpson, and E. Williams, 2003: Measurement of the rates of production and dissipation of turbulent kinetic energy in an energetic tidal flow: Red Wharf Bay revisited. J. Phys. Oceanogr., 33, 1889-1901.

Rosman, J., J. Hench, J. Koseff, and S. Monismith, 2008: Extracting Reynolds stresses from acoustic Doppler current profiler measurements in wave-dominated environments. J. Atmos. Oceanic Technol., 25, 286-306.

Shaw, W., and J. Trowbridge, 2001: The direct estimation of nearbottom turbulent fluxes in the presence of energetic wave motions. J. Atmos. Oceanic Technol., 18, 1540-1557.

Stacey, M., S. Monismith, and J. Burau, 1999a: Measurements of Reynolds stress profiles in unstratified tidal flow. J. Geophys. Res., 104 (C5), 10 933-10 949.

$\longrightarrow,-$, and $-1999 \mathrm{~b}$ : Observations of turbulence in a partially stratified estuary. J. Phys. Oceanogr., 29, 1950-1970.

Trowbridge, J., 1998: On a technique for measurement of turbulent shear stress in the presence of surface waves. J. Atmos. Oceanic Technol., 15, 290-298.

_ zone. J. Phys. Oceanogr., 31, 2403-2417.

Whipple, A., R. Luettich, and H. Seim, 2005: Measurements of Reynolds stress in a wind-driven lagoonal estuary. Ocean Dyn., 56, 169-185, doi:10.1007/s10236-005-0038-x.

Williams, E., and J. Simpson, 2004: Uncertainties in estimates of Reynolds stress and TKE production rate using the ADCP variance method. J. Atmos. Oceanic Technol., 21, 347-357. 